\title{
Photophysics in single light- harvesting complexes II: from micelle to native nanodisks
}

J. Michael Gruber, Stefan Scheidelaar, Henny van Roon, Jan Dekker, J. Antoinette Killian, et al.

J. Michael Gruber, Stefan Scheidelaar, Henny van Roon, Jan P. Dekker, J. Antoinette Killian, Rienk van Grondelle, "Photophysics in single lightharvesting complexes II: from micelle to native nanodisks," Proc. SPIE 9714, Single Molecule Spectroscopy and Superresolution Imaging IX, 97140A (1 March 2016); doi: 10.1117/12.2211588

SPIE. Event: SPIE BiOS, 2016, San Francisco, California, United States 


\title{
Photophysics in single light-harvesting complexes II: from micelle to native nanodisks
}

\author{
J. Michael Gruber ${ }^{a *}$, Stefan Scheidelaar ${ }^{\mathrm{b}}$, Henny van Roon ${ }^{\mathrm{a}}$, Jan P. Dekker ${ }^{\mathrm{a}}$, J. Antoinette Killian ${ }^{\mathrm{b}}$, \\ Rienk van Grondelle ${ }^{\mathrm{a}}$ \\ ${ }^{a}$ Biophysics Section, Department of Physics and Astronomy, Faculty of Sciences, VU University \\ Amsterdam, The Netherlands; ${ }^{b}$ Membrane Biochemistry \& Biophysics, Bijvoet Center for \\ Biomolecular Research, Department of Chemistry, Faculty of Science, Utrecht University
}

\begin{abstract}
Most photosynthetic pigment-protein complexes of algae and higher plants are integral membrane proteins and are thus usually isolated in the presence of detergent to provide a hydrophobic interface and prevent aggregation. It was recently shown that the styrene maleic acid (SMA) copolymer can be used instead to solubilize and isolate protein complexes with their native lipid environment into nanodisk particles. We isolated LHCII complexes in SMA and compared their photophysics with trimeric LHCII complexes in $\beta$-DM detergent micelles to understand the effect of the native environment on the function of light-harvesting antennae. The triplet state kinetics and the calculated relative absorption cross section of single complexes indicate the successful isolation of trimeric complexes in SMA nanodisks, confirming the trimeric structure as the likely native configuration. The survival time of complexes before they photobleach is increased in SMA compared to detergent which might be explained by a stabilizing effect of the co-purified lipids in nanodisks. We furthermore find an unquenched fluorescence lifetime of $3.5 \mathrm{~ns}$ for LHCII in SMA nanodisks which coincides with detergent isolated complexes and notably differs from 2 ns typically found in native thylakoid structures. A large dynamic range of partially quenched complexes both in detergent micelles and lipid nanodisks is demonstrated by correlating the fluorescence lifetime with the intensity and likely reflects the conformational freedom of these complexes. This further supports the hypothesis that fluorescence intermittency is an intrinsic property of LHCII that may be involved in excess energy dissipation in native light-harvesting.
\end{abstract}

Keywords: Light-harvesting complex II (LHCII), Fluorescence lifetime, Styrene maleic acid (SMA), Single molecule spectroscopy (SMS), Absorption cross section, Singlet-Triplet annihilation, Lipid nanodisk, Fluorescence quenching

\section{INTRODUCTION}

Energy transfer in light-harvesting complexes is a highly optimized and efficient process in the primary steps of photosynthesis. ${ }^{1,2}$ The excitation energy of an absorbed photon reaches a reaction center where charge separation and subsequent kinetic trapping of an electron eventually lead to the safe storage of chemical energy. ${ }^{3}$ Minor and major so-called antenna complexes increase the amount of absorbed energy that can be processed by a reaction center. Oxygenic photosynthesis in green plants and algae is driven by photosystem II (PSII) and photosystem I (PSI) that consist of reaction centers and associated antenna complexes. The major light-harvesting complex II (LHCII) is a trimeric antenna complex of both photosystems $\mathrm{s}^{4,5}$ and is densely packed with chlorophyll (Chl) and carotenoid (Car) pigments. One monomeric sub-unit contains eight Chl $a$, six $\mathrm{Chl} b$, two luteins (Lut) and one neoxanthin. ${ }^{6}$ Each monomer within the trimeric complex can also bind either violaxanthin or zeaxanthin depending on the light stress conditions of spinach leaves before protein isolation. ${ }^{7}$ Apart from its main function to harvest sunlight, LHCII is also considered to be involved in a photo-protective mechanism called non-photochemical quenching (NPQ) which regulates the amount of excitation energy that reaches the reaction center in high light conditions. ${ }^{8-11}$ The excess energy is thereby safely dissipated as heat and does not lead to the destruction of reaction centers (photoinhibition). There are multiple hypotheses on the precise molecular mechanism but it is hard to relate the results found in in vitro systems to the actual mechanisms present in in vivo conditions. One of the problems is the loss of the native lipid environment when studying isolated complexes in detergent. It has been shown that different lipids can have either a stimulating or inhibiting influence on the ability of LHCII monomers to form trimeric or crystalline structures. ${ }^{12}$ In our experiments we utilize the SMA method to completely avoid the usage of detergent and instead

\footnotetext{
*j.m.gruber@vu.nl
} 
obtain single light-harvesting complexes in their native lipid environment. ${ }^{13-15}$ We use the advantage of single molecule spectroscopy (SMS) to distinguish the trimeric LHCII complexes from possible sub-populations of monomeric complexes and PSII core particles. Apart from the absolute fluorescence intensity of single particles we also look at the inherent photophysics of LHCII complexes, namely the formation of Car triplets and subsequent Singlet-Triplet (S-T) annihilation under strong light conditions. This last mechanism depends among other parameters on the absorption cross section of the complex, the functional energy transfer in the complex and the triplet kinetics of the present Cars and is therefore a good approach to investigate the composition of the measured complexes. About a third of the absorbed photons will form Chl $a$ triplet states that are quickly quenched by Cars to form Car triplet states. This is an essential photo-protective mechanism to inhibit the formation of harmful oxygen radicals from energetically higher Chl triplet states. ${ }^{16}$ At high photon absorption rates of more than $3 \cdot 10^{5} \mathrm{~s}^{-1}$ there is a significant chance for the coincident presence of a singlet excitation and a Car triplet state which results in a rapid dissipation of the singlet excitation to heat, a process called S-T annihilation.

\section{MATERIALS AND METHODS}

\subsection{Isolation of LHCII in SMA nanodisks}

Thylakoid membranes of spinach were prepared as described previously. ${ }^{17}$ These membranes were mixed with the SMA polymer X25010 in a buffer (20 mM Hepes, $100 \mathrm{mM} \mathrm{NaCl}, \mathrm{pH} 7.5)$, incubated at room temperature for one hour and then centrifuged for 15 minutes at $17500 \mathrm{~g}$ at $4^{\circ} \mathrm{C}$ in an eppendorf table centrifuge to separate the SMA nanodisks from non-solubilized membranes and bigger aggregates. The supernatant containing the solubilized SMA nanodisks was then fractionated by ultracentrifugation in a $0.6 \mathrm{M}$ sucrose gradient (prepared by the freeze-thaw method) at a speed of 150000 $\mathrm{g}$ for 17 hours at $4^{\circ} \mathrm{C}$. The sample was highly diluted in a buffer solution ( $30 \mathrm{mM}$ Hepes, $\mathrm{pH}$ 7.6) and then immobilized on a PLL (poly-L-Lysine) coated cover glass. ${ }^{18}$ The concentration was determined empirically to achieve a surface density of about 10 complexes per $100 \mu \mathrm{m}^{2}$. Oxygen was removed from the closed sample chamber with an enzymatic scavenging mix comprised of $200 \mu \mathrm{g} / \mathrm{ml}$ glucose oxidase, $35 \mu \mathrm{g} / \mathrm{ml}$ catalase and $7.5 \mathrm{mg} / \mathrm{ml}$ glucose. This step was necessary to avoid fast photobleaching via reactive oxygen species. The monomeric and trimeric LHCII control samples obtained from detergent isolation were diluted in a buffer solution containing $0.03 \%(\mathrm{w} / \mathrm{v}) \mathrm{n}$-Dodecyl $\beta$-D-maltoside $(\beta$-DM).

\subsection{Single molecule spectroscopy}

The fluorescence intensity, spectrum and lifetime experiments were performed at room temperature on a confocal fluorescence setup as described earlier. ${ }^{18,19}$ In short, the complexes were excited at $633 \mathrm{~nm}$ with an excitation intensity of $75 \mathrm{~W} / \mathrm{cm}^{2}$ by a pulsed laser with $200 \mathrm{fs}$ pulse length and $76 \mathrm{MHz}$ repetition rate. Near-circularly polarized light was used to avoid the orientation dependence of randomly oriented complexes. The fluorescence intensity was detected by a single photon avalanche photo-diode (Micro Photon Devices, PDM series). Alternatively the fluorescence spectrum was detected via a grating and a CCD (Roper Scientific, Spec10:100BR) with an integration time of one second. A time-correlated single photon counting (TCSPC) device (PicoHarp 300, PicoQuant) allowed to acquire both the absolute and relative (triggered by the pulsed laser excitation) arrival time of the detected photons. In order to investigate the composition and size of the protein complexes we utilize the following methods to determine their photophysics and relative absorption cross section.

\subsubsection{S-T annihilation kinetics}

The experimental method to extract the $\mu$ s fluorescence intensity kinetics via pulse wave excitation by means of an acoustooptic modulator (MT350, Acousto-Optic Devices) is described in detail elsewhere. ${ }^{19}$ It allows to follow the accumulation and decay of Car triplet states via the influence of S-T annihilation on the resulting fluorescence intensity. These Car triplet states are rapidly $(<1 \mathrm{ps})$ populated from Chl triplet states via triplet energy transfer and the intrinsic triplet yield of Chl in a protein environment is about $30 \% .{ }^{20}$

\subsubsection{Fluorescence lifetime fitting}

The fluorescence lifetime of individual stable intensity levels was extracted from the resulting fluorescence decay histograms by fitting them with a reconvolution algorithm in the software FluoFit (PicoQuant). The intensity levels were thereby determined by a Matlab script described in Krüger et al., ${ }^{21}$ the binning time of the decay histograms was 4 ps and the instrument response function (IRF) had a FWHM of about $38 \mathrm{ps}$. The fluorescence decay of LHCII in single molecule conditions has to be fitted with two decay component due to the presence of S-T annihilation as described elsewhere. ${ }^{19}$ The slower component $\tau_{\text {slow }}$ between roughly $100 \mathrm{ps}$ and $3.5 \mathrm{~ns}$ represent the real overall singlet excited state decay without 
annihilation. It is well separated from the width of the IRF and therefore accurately determined by a reconvolution fit. The fast component $\tau_{\text {fast }}=36$ ps corresponds to S-T annihilation events and its amplitude $A_{\text {fast }}$ is not that easily fitted due to the small number of detected photons. The amplitude ratio $R_{\text {fast }}=\frac{A_{\text {fast }}}{A_{\text {fast }}+A_{\text {slow }}}$ is up to $50 \%$ at an excitation intensity of $75 \mathrm{~W} / \mathrm{cm}^{2}$. This results in an up to a hundred times smaller fluorescence yield than the slow component.

\subsubsection{Correction of the fast lifetime amplitude}

Instead of using the often misfitted value for the amplitude of the fast lifetime component, one can also calculate the expected amount of S-T annihilation based on previously published theoretical results. ${ }^{19,22}$ A change in the fluorescence lifetime $\tau_{\text {slow }}$ due to any other quenching mechanism than annihilation is directly proportional to the triplet yield $\varphi_{I S C}=$ $\tau_{\text {slow }} \cdot k_{I S C}$ and therefore changes the saturation intensity $I_{s a t}$ of the fluorescence rate $k_{f}$ at a constant excitation intensity $I_{e}$ :

$$
k_{f}=\frac{I_{e}}{I_{e}+I_{\text {sat }}} \cdot k_{f}^{\max } \quad \text { with } \quad I_{\text {sat }} \propto \frac{1}{\tau_{\text {slow }}}
$$

In other words, quenched complexes have a lower probability to contain a triplet state and will therefore have a smaller amplitude fraction of annihilated excitations. The value for $k_{f}^{\max }$ and the saturation lifetime $\tau_{\text {sat }}$ depend on the excitation intensity $I_{e}$ but can be obtained experimentally and solving for the fluorescence rate yields

$$
k_{f}\left(\tau_{\text {slow }}\right)=\frac{\tau_{\text {slow }}}{\tau_{\text {slow }}+\tau_{\text {sat }}} \cdot k_{f}^{\max }
$$

The ratio $\frac{k_{f}}{k_{f}^{\max }} \approx R_{f a s t}$ can now be used to estimate the amplitude of the fast lifetime component and the resulting values of the average lifetime and the relative absorption cross section.

\subsubsection{Relative absorption cross section}

The estimation of the relative absorption cross section $\sigma$ can be obtained from the complimentary information on the fluorescence intensity and the two lifetime amplitudes $A_{i}$ of the fluorescence decay. A similar approach was described earlier to quantify the fluorescence brightness of single molecules. ${ }^{23}$ The rate of absorbing photons $k_{a b s}$ can be calculated by Eq. 3 and can be used to approximate the absorption cross section at a photon energy $E_{\text {photon }}$ and an excitation intensity $I_{e}$ :

$$
\begin{gathered}
k_{a b s}=\frac{1}{\eta \cdot t_{a c q}} \cdot \sum_{i} \frac{A_{i} \cdot \tau_{i}}{t_{b i n} \cdot \varphi_{i}}=\frac{\sum_{i} A_{i}}{\eta \cdot t_{a c q} \cdot t_{b i n} \cdot k_{f}} \\
\sigma=\frac{k_{a b s}}{I_{e} / E_{\text {Photon }}}
\end{gathered}
$$

$\eta$ is the relative overall detection efficiency of the setup, $t_{a c q}$ is the acquisition time of the decay histogram, $t_{b i n}$ is the binning time of the fluorescence decay, $\tau_{i}$ are the two lifetime components and $\varphi_{i}$ the associated fluorescence yields of one decay component. A typical radiative rate of $k_{f}=16 \mathrm{~ns}$ was assumed to be constant and the same between different samples. ${ }^{24,25}$ The detection efficiency can only be estimated (about 5\%) but stays constant between two measurements. Systematic errors can arise from changes in the radiative rate $k_{f}$ and misfits of the decay components including missing very fast decay processes ( $<5$ ps) can also lead to errors in the calculated absorption cross section.

The following data for the absorption cross section and fluorescence lifetime distributions was obtained from data-sets of about 200 complexes with an acquisition time of 120 seconds each. The fluorescence spectrum of each was measured with an integration time of one second. 

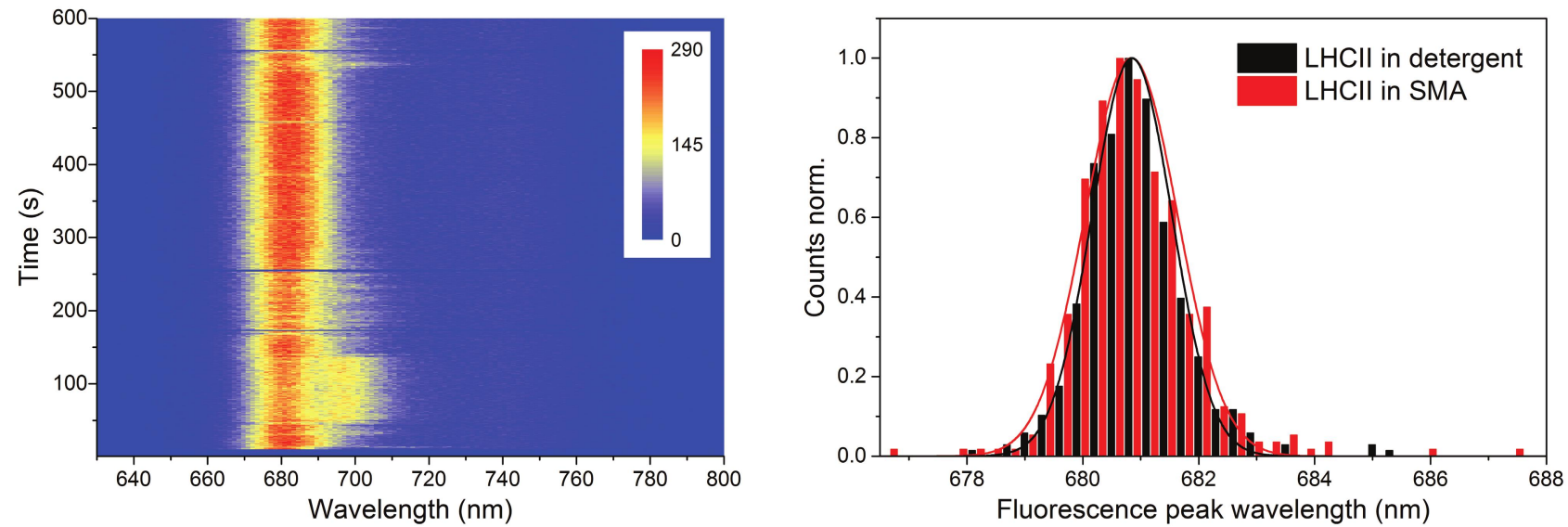

Figure 1: Left: Contour map of the fluorescence emission of a single complex in a SMA nanodisk. The color code represents the number of photon counts within one second integration time and a wavelength bin of about $1.25 \mathrm{~nm}$. Right: Fluorescence peak distribution of the main emission peak at about $681 \mathrm{~nm}$ of LHCII trimers in detergent and SMA nanodisks. Each distributions contains about 400 spectra. The colored lines show the fitted normal distributions and indicate a slightly wider distribution for LHCII in SMA nanodisks.

\section{EXPERIMENTAL RESULTS}

Our comparison of complexes in detergent and SMA starts with the fluorescence emission spectrum. The left graph in Fig. 1 shows the time evolution of the fluorescence spectrum of an exemplary particle in a SMA nanodisk. We should note that the depicted example is only one realization of the stochastic nature of blinking and off-states with dwell-times up to minutes, i.e. the period of time a complex remains in a dark non-fluorescent state, are not uncommon. These blinking events are a characteristic feature of a single particle with a well connected electronic excited state manifold. The main emission peak is at about $681 \mathrm{~nm}$ and this particular example was chosen to also illustrate the appearance of a second, red shifted emission peak at about $700 \mathrm{~nm}$. These red shifted peaks appear with the same probability of about 3-5\% as in detergent isolated LHCII trimers ${ }^{18}$ and therefore most likely reflect an intrinsic property of LHCII. The right graph in Fig. 1 illustrates the peak wavelength distribution of the main $681 \mathrm{~nm}$ emission band for both the SMA sample and the detergent control. The distributions overlap which indicates that the emission originates from Chl pigments with a very similar electronic structure, at least for the equilibrated terminal emitters. The width of that distribution is furthermore a good indicator of the amount of static disorder exhibited by the sample. The FWHM of the distribution for the SMA sample is slightly bigger $(1.9 \mathrm{~nm} \pm 0.1 \mathrm{~nm})$ than the control sample in detergent $(1.6 \pm 0.1 \mathrm{~nm})$.

\subsection{S-T annihilation kinetics}

The spectral characterization in Fig. 1 is not sufficient to identify the complexes within the SMA nanodisks as trimeric LHCII complexes. The goal is to distinguish them from monomeric light-harvesting complexes (LHC) and LHC aggregates. In order to get an idea about the photophysics and indirectly the composition of the complexes we utilized a pulse wave excitation method described earlier. ${ }^{19}$ This effectively allows us to observe the amount and accumulation of Car triplet states within each of the complexes. In short, by modulating the excitation pattern with a pulse wave train (frequency $5 \mathrm{kHz}$ and duty cycle 0.9 ) the Car triplet states decay to the ground state in the time window between two subsequent pulse waves and the fluorescence intensity within one pulse wave reflects the accumulation of those Car triplet states. The intensity time traces shown in Fig. 2 are acquired by histogramming over many modulation cycles. The resulting amplitude and accumulation rates theoretically depend among other things on the excitation density and therefore on the used excitation intensity and the absorption cross section of the complexes. Fig. 2 illustrates the accumulation of Car triplet states for both the complexes in SMA nanodisks and an LHCII control in detergent for multiple excitation intensities. The kinetics exhibit a good agreement at identical excitation intensities. This implies that the complexes in SMA and in detergent contain about the same amount of $\mathrm{Chl}$ and that the S-T annihilation kinetics arise from a similar composition of pigments. 


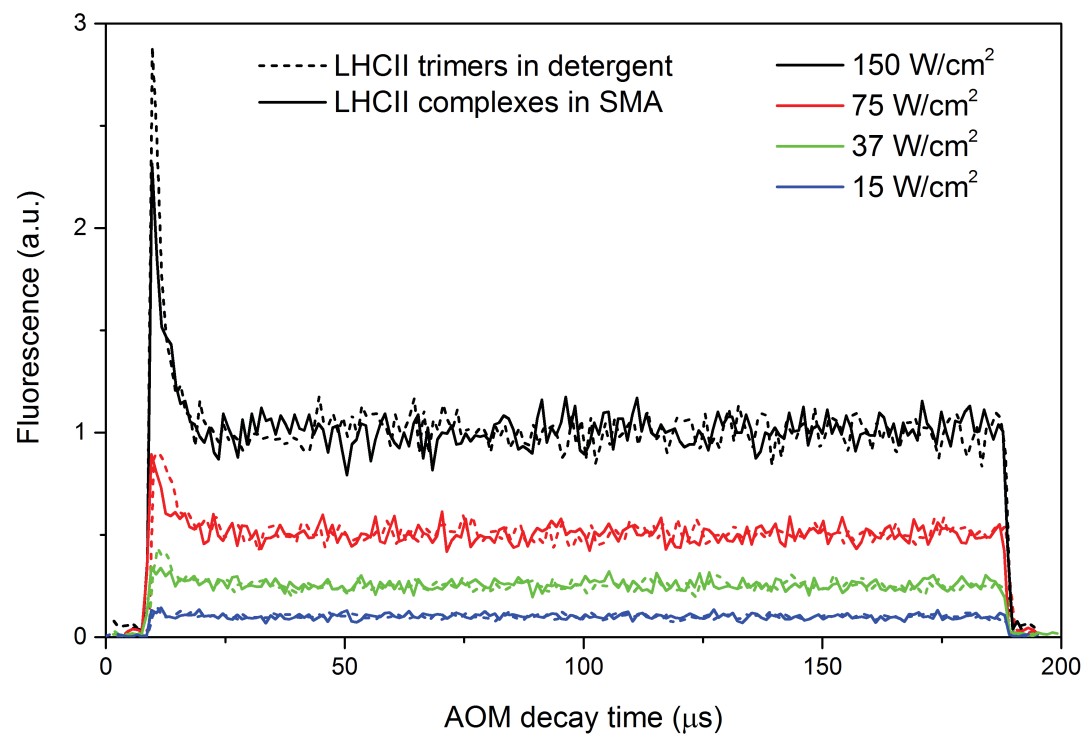

Figure 2: Fluorescence intensity histograms for pulse wave excitation with a frequency of $5 \mathrm{kHz}$ and a duty cycle of 0.9 . The steady state plateaus of the traces are scaled to the excitation intensity and normalized to the highest excitation intensity. The graph represents the change in fluorescence within one modulation cycle and clearly illustrates the accumulation of Car triplets which quench the fluorescence via S-T annihilation. Both the amount and the accumulation rate of triplet states depend on the excitation intensity but also on the size and connectivity of the sample. The traces of the detergent sample (dashed lines) and the SMA sample (solid line) are very similar for varying excitation intensities (different colors).

\subsection{Relative absorption cross section}

To further quantify the information about the size of the light-harvesting complex we estimated the relative absorption cross section. As the utilized excitation wavelength is $633 \mathrm{~nm}$ and the electromagnetic field is near-circular polarized, this method yields a good approximation of the amount of Chl present in the confocal excitation volume. This can also be experimentally validated with control samples of monomeric (black bars) and trimeric (green bars) LHC complexes in detergent as shown in Fig. 3. The transparently colored bars represent the values calculated from the fitted lifetime amplitudes and show relatively broad and asymmetric distributions due to fitting errors in the fast lifetime amplitude. As the annihilation kinetics described earlier are similar for both samples we chose to substitute the fast lifetime amplitude with the expected amount of annihilation as described in the Materials and Methods section. This results in narrower and symmetric distributions. The SMA sample is clearly in the correct range for trimeric complexes but is slightly shifted to larger values.

\subsection{Fluorescence lifetime}

Finally, we can use the measured singlet excited state decay to plot the fluorescence lifetime against the detected fluorescence intensity. In order to do that, fluorescence intensity traces (acquisition time of 120 seconds) were first split up into time intervals of constant intensity and the corresponding excited state decay histograms were subsequently fitted with a two-exponential reconvolution model to extract the amplitudes and decay rates. Two exponents were needed to account for the significant amount of S-T annihilation (about $50 \%$ at $75 \mathrm{~W} / \mathrm{cm}^{2}$ ), which contributes a lifetime component of about 36 ps. The two graphs on the left side of Fig. 4 represent data from LHCII complexes in SMA nanodisks, whereas the right two graphs correspond to LHCII trimers in detergent. The two top graphs depict the correlation between the amplitude averaged fluorescence lifetime $\left(\tau_{a v g}=\frac{A_{1} \cdot \tau_{1}+A_{2} \cdot \tau_{2}}{A_{1}+A_{2}}\right)$ and the fluorescence intensity. The color-coded pixel represent the relative dwell-time of complexes in the associated fluorescence intensity (50 cps binning) and average fluorescence lifetime (100 ps binning) range. The black dots in the top graphs depict the calculated average lifetime values based on the fitted amplitudes and again show a relatively wide spread distribution. Using the estimated annihilation contribution to calculate the average lifetime results in narrower distributions. Both samples show a clear linear relationship as indicated by the 


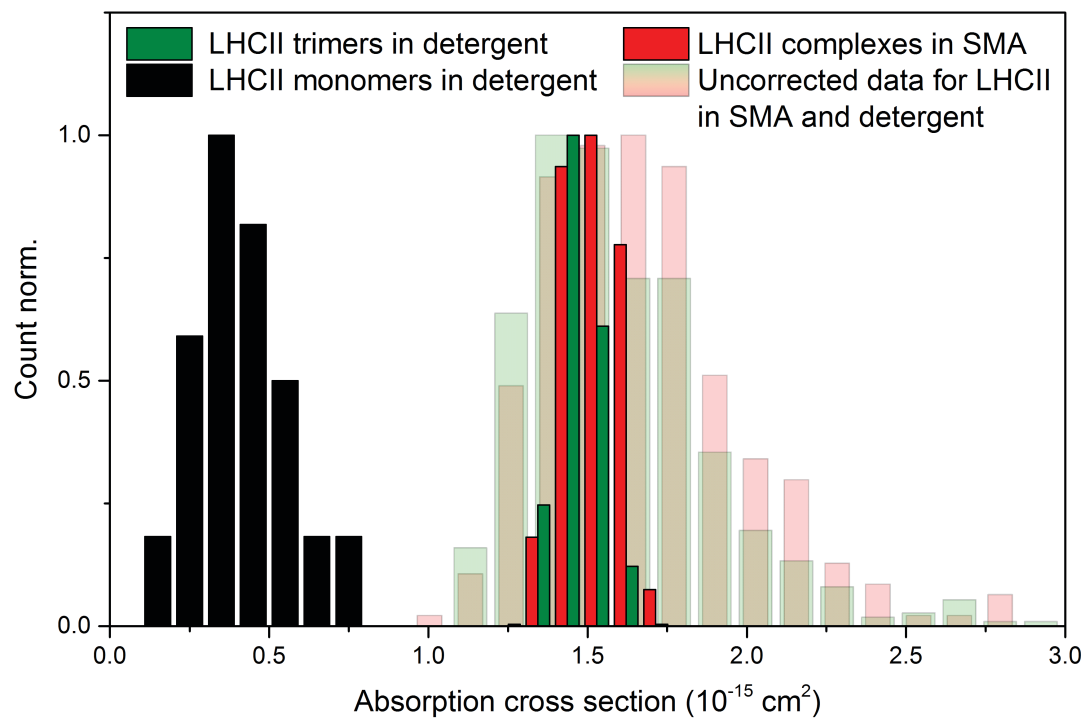

Figure 3: Comparison of the obtained absorption cross section histograms for LHCII trimers in detergent (green) and complexes in SMA (red). The solid bars represent values obtained with an estimated amount of annihilation. Control measurements on LHC monomers are shown in black. The shaded bars represent the values obtained from the fitted amplitude of the annihilation component and illustrate the large fitting error due to the small amount of photon counts.

dashed line. However, the average lifetime values contain the contribution from S-T annihilation and therefore cannot be compared to ensemble measurements typically done under annihilation free conditions. Plotting only the slow lifetime component in the two bottom graphs in Fig. 4 results in lifetimes values that correspond to annihilation free conditions. The fluorescence intensity is still affected by annihilation, which explains the resulting curved shape of the distribution. In line with the preceding experiments, the results for LHCII in SMA nanodisks do not differ significantly from the detergent control. Importantly, the main unquenched state of LHCII complexes in the lipid environment has a lifetime in the range of about 3 to $3.5 \mathrm{~ns}$, similar to the reported lifetime of the detergent isolated LHCII trimers. ${ }^{19,26-28}$ The black dashed lines in the two bottom graphs show the theoretical dependence described by Eq. 2.

\section{DISCUSSION AND CONCLUSIONS}

The goal of this research project was to obtain information on the behavior of single light-harvesting antennae in a nativelike environment and to compare them against detergent isolated complexes. With the SMA polymer we were able to excise trimeric LHCII complexes and their surrounding lipids directly out of the thylakoid membrane. Previous ensemble measurements on light-harvesting complexes in a lipid environment were performed by incorporation of detergent isolated complexes back into an artificially prepared lipid environment, e.g. liposomes and lipid nanodisks prepared via membrane scaffolding proteins (MSP). ${ }^{26,29-31}$ For proteoliposomes this often resulted in quenched lifetime of less than 2 ns. MSP nanodisks instead, that due to their size can contain only single complexes, were reported to retain a slow lifetime component of about $3.5 \mathrm{~ns}^{31}$ However, it was unclear whether these MSP nanodisks contain LHCII in a monomeric or trimeric state. We indeed find and confirm the same slow lifetime component of more than $3 \mathrm{~ns}$ in single complexes. This supports the hypothesis that the quenched fluorescence lifetime of about 2 ns typically found in native thylakoid structures ${ }^{32}$ or liposomes is more likely caused by protein-protein interactions than by the lipid environment. We furthermore show a wide distribution of intermediate states with their associated quenched lifetimes. This distribution arises most likely from different conformational states of the protein matrix surrounding and determining the excitonic manifold of the comprising pigments. The average lifetime distributions shown in Fig. 4 are clearly linearly proportional to the fluorescence intensity which is in agreement with the theoretical expectation of a well-connected and equilibrated complex where the overall excited state decay is given by the sum of all possible decay rates. ${ }^{33}$ The good agreement of the theoretically expected relation between the slow fluorescence lifetime and the fluorescence intensity with the experimental data shown in the 

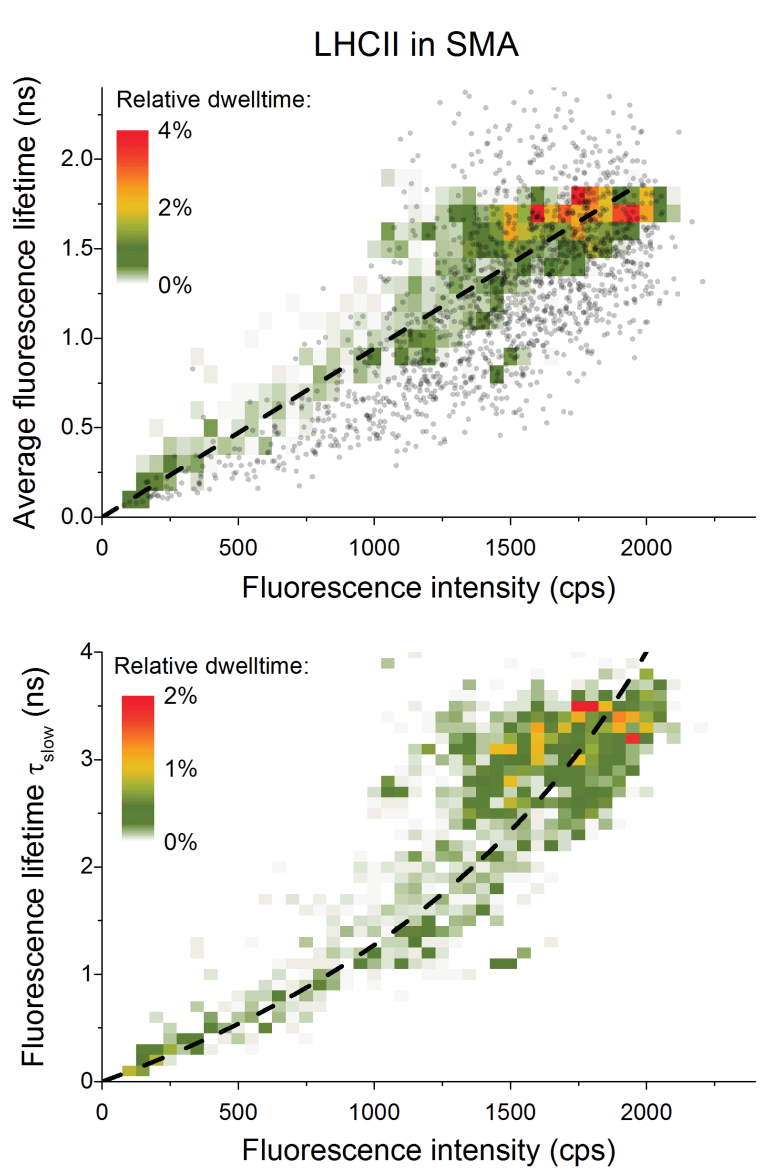

\section{LHCII in detergent}
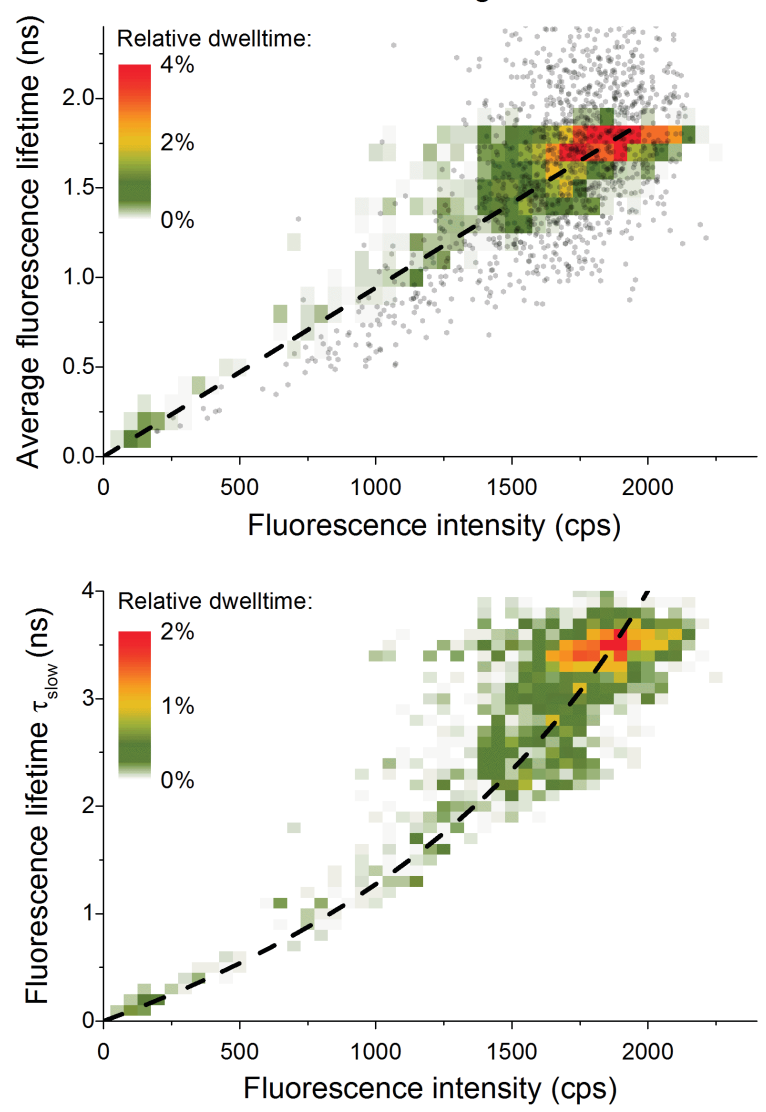

Figure 4: Comparison of the fluorescence lifetime characteristic of LHCII trimers in detergent (right) and in SMA nanodisks (left). The two top graphs show the amplitude averaged fluorescence lifetime plotted against the fluorescence intensity. The amplitude of the fast lifetime component was estimated by the expected amount of annihilation. The color code represents the overall dwell-time probability of complexes within an intensity bin of $50 \mathrm{cps}$ and a lifetime bin of $100 \mathrm{ps}$. The two bottom graphs depict the fluorescence lifetime $\tau_{\text {slow }}$ without the contribution of S-T annihilation on the y-axis while the measured fluorescence intensity on the x-axis is due to annihilation still saturating at larger lifetime values. The black dashed lines in the top graphs illustrate the linear relation ship between average lifetime and fluorescence intensity. In the bottom graphs these black lines illustrate the theoretically calculated relation between the slow lifetime component and the fluorescence intensity (Eq. 2). The transparent black dots in the top graphs illustrate the fitted values from individual intensity levels without dwell-time weighting and without corrections of the amplitude of the fast annihilation component as described in the Materials and Methods section. 
lower panels of Fig. 4 validates the applicability of our method to estimate the amount of S-T annihilation. The fitted values without correction of the average lifetime (see Fig. 4, black dots in the upper right panel) clearly show the deviations in intermediate states due to the misfit of the fast lifetime component. The average lifetime at the unquenched and strongly quenched level is nevertheless similar. In a previous study on single detergent isolated LHCII complexes, deviating intermediate states were shown with an unquenched slow lifetime component but with a lower fluorescence intensity ${ }^{27}$ Here we also observe a small shoulder towards lower intensities but not as prominent as in Schlau-Cohen et al. ${ }^{27}$ It is furthermore possible that small lateral or axial drifts of the confocal spot during the time scale of two minutes lead to a systematic error towards smaller intensities without a change in the slow lifetime component. Instead of well-defined states we observe a distribution of quenched states all along the trajectory of the overall excited state lifetime. This is even more pronounced in the case of LHCII trimers in the native lipid environment. This can be interpreted as the presence of an equilibrated quencher with a slow variable quenching rate or as the transfer limited population of a strong quencher with inverted transfer kinetics. ${ }^{10,34,35}$ Our experiments do not allow to distinguish those possibilities but the broad range of quenching rates and dwell-times up to seconds do suggest that the mechanism is indeed influenced by static disorder. ${ }^{36}$ The fact that we observe fluorescence intermittency with a well-defined off-state (dark state) might also be caused by the fact that any underlying distribution of quenching rates that lead to an overall excited state lifetime of less than about 100 ps cannot be resolved in single molecule experiments and will just appear as a dark state. Some of these strongly quenched states do not show up in Fig. 4 due to the lack of fluorescence photons.

SMS furthermore allows us to selectively analyze single complexes and verify their trimeric state as illustrated in the previous section. Another advantage of SMA nanodisks is that they are assembled straight from the thylakoid membrane, i.e. complexes and their immediate surroundings are literally 'cut' out of the membrane. Possible protein deformation or loss of cofactors in an artificial incorporation process can therefore be avoided. There is indeed a small shift of the absorption cross section of LHCII trimers in SMA nanodisks towards larger values which can be interpreted as the presence of additional pigments. The stability of complexes can be enhanced in their native lipid environment compared to detergent isolation as has already been shown for bacterial reaction centers in SMA nanodisks. ${ }^{14}$ Lipids are essential to trimer formation and NMR experiments also show the presence of co-purified lipids in detergent isolated complexes. ${ }^{31,37}$ LHCII complexes in SMA nanodisks are remarkably stable as illustrated by the exemplary complex on the left side in Fig. 1 that is still not photobleached or degraded after ten minutes of measurement. More than $40 \%$ of about 50 complexes in SMA exhibited unquenched fluorescence emission after 10 minutes of measurement in contrast to the characteristic survival rate of about one to two minutes for LHCII in detergent micelles. ${ }^{18,21}$ This suggests that the lipid environment either lowers the formation of statically and strongly quenched conformational states or reduces the rate of photoinhibition. These results are in line with a detailed study on the increased stability of bacterial reaction centers in SMA complexes. ${ }^{14}$

The conformational space of protein folding is another important parameter that influences the connected electronic and vibronic excited state manifold of the pigments. Our fluorescence lifetime measurements indicate that there is a difference in the population of intermediately quenched states, possibly reflecting a variation in the conformational freedom of LHCII in a lipid environment. Recent Molecular Dynamics simulations for example support the hypothesis of a highly flexible protein conformation in a lipid environment. ${ }^{38}$ The same trend is observed in the spectral fluorescence peak distribution with a wider distribution for the complexes in the SMA nanodisks.

Overall we can conclude that LHCII complexes are more stable in SMA nanodisks than in detergent micelles but there are no major differences in their fluorescence features. This justifies the extensive use of detergent to purify and study isolated photosynthetic protein complexes. ${ }^{39}$ Nevertheless, small differences in the native lipid environment might very well play a role in the regulated function of energy dissipation that potentially make use of the intrinsic conformational space of these light-harvesting complexes.

\section{ACKNOWLEDGMENTS}

The authors kindly thank Pengqi Xu of the group of Prof. Roberta Croce (VU Amsterdam) for helpful biochemical advice and Tjaart Krüger (University of Pretoria) for fruitful discussions. J.M.G. and R.v.G. were supported by the VU University and by an Advanced Investigator grant from the European Research Council (no. 267333, PHOTPROT) to R.v.G.; R.v.G. was also supported by the Nederlandse Organisatie voor Wetenschappelijk Onderzoek, Council of Chemical Sciences (NWO-CW) via a TOP-grant (700.58.305), and by the EU FP7 project PAPETS (GA 323901). R.v.G. gratefully acknowledges his Academy Professor grant from the Netherlands Royal Academy of Sciences (KNAW). 


\section{REFERENCES}

[1] Croce, R. and van Amerongen, H., "Natural strategies for photosynthetic light harvesting.," Nature chemical biology 10(7), 492-501 (2014).

[2] van Grondelle, R., Dekker, J. P., Gillbro, T., and Sundstrom, V., "Energy transfer and trapping in photosynthesis," Biochimica et Biophysica Acta (BBA) - Bioenergetics 1187(1), 1-65 (1994).

[3] Blankenship, R. E., [Molecular Mechanisms of Photosynthesis], John Wiley \& Sons, 103-135 (2013).

[4] Allen, J. F., "Protein phosphorylation in regulation of photosynthesis.," Biochimica et Biophysica Acta (BBA) Bioenergetics 1098(3), 275-335 (1992).

[5] Kouril, R., Zygadlo, A., Arteni, A. A., de Wit, C. D., Dekker, J. P., Jensen, P. E., Scheller, H. V., and Boekema, E. J., "Structural characterization of a complex of photosystem I and light-harvesting complex II of Arabidopsis thaliana.," Biochemistry 44(33), 10935-40 (2005).

[6] Liu, Z., Yan, H., Wang, K., Kuang, T., Zhang, J., Gui, L., An, X., and Chang, W., "Crystal structure of spinach major light-harvesting complex at 2.72 A resolution.," Nature 428(6980), 287-292 (2004).

[7] Ruban, A. V., Lee, P. J., Wentworth, M., Young, A. J., and Horton, P., "Determination of the Stoichiometry and Strength of Binding of Xanthophylls to the Photosystem II Light Harvesting Complexes," Journal of Biological Chemistry 274(15), 10458-10465 (1999).

[8] Horton, P. and Ruban, A., "Molecular design of the photosystem II light-harvesting antenna: Photosynthesis and photoprotection," Journal of Experimental Botany 56(411), 365-373 (2005).

[9] Ruban, A. V., Johnson, M. P., and Duffy, C. D. P., "The photoprotective molecular switch in the photosystem II antenna," Biochimica et Biophysica Acta (BBA) - Bioenergetics 1817(1), 167-181 (2012).

[10] Ruban, A. V., Berera, R., Ilioaia, C., van Stokkum, I. H., Kennis, J. T. M., Pascal, A. a., van Amerongen, H., Robert, B., Horton, P., and van Grondelle, R., "Identification of a mechanism of photoprotective energy dissipation in higher plants.," Nature 450(7169), 575-578 (2007).

[11] Standfuss, J., Terwisscha van Scheltinga, A. C., Lamborghini, M., and Kühlbrandt, W., "Mechanisms of photoprotection and nonphotochemical quenching in pea light-harvesting complex at 2.5 A resolution.," The EMBO Journal 24(5), 919-28 (2005).

[12] Reinsberg, D., Booth, P. J., Jegerschöld, C., Khoo, B. J., and Paulsen, H., "Folding, Assembly, and Stability of the Major Light-Harvesting Complex of Higher Plants, LHCII, in the Presence of Native Lipids," Biochemistry 39(46), 14305-14313 (2000).

[13] Scheidelaar, S., Koorengevel, M., Pardo, J., Meeldijk, J., Breukink, E., and Killian, J., "Molecular Model for the Solubilization of Membranes into Nanodisks by Styrene Maleic Acid Copolymers," Biophysical Journal 108(2), 279-290 (2015).

[14] Swainsbury, D. J. K., Scheidelaar, S., van Grondelle, R., Killian, J. A., and Jones, M. R., "Bacterial reaction centers purified with styrene maleic acid copolymer retain native membrane functional properties and display enhanced stability.," Angewandte Chemie (International ed. in English) 53(44), 11803-7 (2014).

[15] Dörr, J. M., Scheidelaar, S., Koorengevel, M. C., Dominguez, J. J., Schäfer, M., van Walree, C. A., and Killian, J. A., "The styrene-maleic acid copolymer: a versatile tool in membrane research," European Biophysics Journal 45(1), 3-21 (2016).

[16] Yasushi, K., "New trends in photobiology," Journal of Photochemistry and Photobiology B: Biology 9(3-4), 265280 (1991).

[17] van Roon, H., Van Breemen, J. F. L., De Weerd, F. L., Dekker, J. P., and Boekema, E. J., "Solubilization of green plant thylakoid membranes with n-dodecyl- $\alpha$,D-maltoside. Implications for the structural organization of the Photosystem II, Photosystem I, ATP synthase and cytochrome b6f complexes," Photosynthesis Research 64(23), 155-166 (2000).

[18] Krüger, T., Novoderezhkin, V., Ilioaia, C., and Van Grondelle, R., "Fluorescence spectral dynamics of single LHCII trimers," Biophysical Journal 98(12), 3093-3101 (2010).

[19] Gruber, J. M., Chmeliov, J., Krüger, T. P. J., Valkunas, L., and van Grondelle, R., "Singlet - triplet annihilation in single LHCII complexes," Phys. Chem. Chem. Phys. 17(30), 19844-19853 (2015).

[20] Kramer, H. and Mathis, P., "Quantum yield and rate of formation of the carotenoid triplet state in photosynthetic structures.," Biochimica et Biophysica Acta (BBA) - Bioenergetics 593(2), 319-329 (1980). 
[21] Krüger, T. P. J., Ilioaia, C., and van Grondelle, R., "Fluorescence intermittency from the main plant lightharvesting complex: resolving shifts between intensity levels.," The Journal of Physical Chemistry B 115(18), 5071-82 (2011).

[22] Krüger, T. P. J., [From disorder to order: The functional flexibility of single plant light-harvesting complexes], Vrije Universiteit, Amsterdam, 38-39 (2011).

[23] Tian, Y., Halle, J., Wojdyr, M., Sahoo, D., and Scheblykin, I. G., "Quantitative measurement of fluorescence brightness of single molecules," Methods and Applications in Fluorescence 2(3), 035003 (2014).

[24] Palacios, M. A., de Weerd, F. L., Ihalainen, J. A., van Grondelle, R., and van Amerongen, H., "Superradiance and Exciton (De)localization in Light-Harvesting Complex II from Green Plants," The Journal of Physical Chemistry B 106(22), 5782-5787 (2002).

[25] Bennett, D. I. G., Amarnath, K., and Fleming, G. R., "A structure-based model of energy transfer reveals the principles of light harvesting in photosystem II supercomplexes.," Journal of the American Chemical Society 135(24), 9164-73 (2013).

[26] Moya, I., Silvestri, M., Vallon, O., Cinque, G., and Bassi, R., "Time-resolved fluorescence analysis of the photosystem II antenna proteins in detergent micelles and liposomes," Biochemistry 40(42), 12552-12561 (2001).

[27] Schlau-Cohen, G. S., Yang, H.-Y., Krüger, T. P. J., Xu, P., Gwizdala, M., van Grondelle, R., Croce, R., and Moerner, W. E., "Single-Molecule Identification of Quenched and Unquenched States of LHCII," The Journal of Physical Chemistry Letters 6, 860-867 (2015).

[28] van Oort, B., van Hoek, A., Ruban, A. V., and van Amerongen, H., "Aggregation of Light-Harvesting Complex II leads to formation of efficient excitation energy traps in monomeric and trimeric complexes," FEBS Letters 581(18), 3528-3532 (2007).

[29] Iwaszko, E., Wardak, A., Krupa, Z., and Gruszecki, W. I., "Ion transport across model lipid membranes containing light-harvesting complex II: an effect of light," Journal of Photochemistry and Photobiology B: Biology 74(1), 1321 (2004).

[30] Schaller, S., Latowski, D., Jemiola-Rzeminska, M., Wilhelm, C., Strzalka, K., and Goss, R., "The main thylakoid membrane lipid monogalactosyldiacylglycerol (MGDG) promotes the de-epoxidation of violaxanthin associated with the light-harvesting complex of photosystem II (LHCII).," Biochimica et Biophysica Acta (BBA) - Bioenergetics 1797(3), 414-24 (2010).

[31] Pandit, A., Shirzad-Wasei, N., Wlodarczyk, L. M., van Roon, H., Boekema, E. J., Dekker, J. P., and de Grip, W. J., "Assembly of the Major Light-Harvesting Complex II in Lipid Nanodiscs," Biophysical Journal 101(10), 2507-2515 (2011).

[32] Tian, L., Dinc, E., and Croce, R., "LHCII Populations in Different Quenching States Are Present in the Thylakoid Membranes in a Ratio that Depends on the Light Conditions," The Journal of Physical Chemistry Letters 6(12), 2339-2344 (2015).

[33] Berezin, M. Y. and Achilefu, S., "Fluorescence lifetime measurements and biological imaging.," Chemical reviews 110(5), 2641-84 (2010).

[34] Duffy, C. D. P., Chmeliov, J., Macernis, M., Sulskus, J., Valkunas, L., and Ruban, A. V., "Modeling of fluorescence quenching by lutein in the plant light-harvesting complex LHCII.," The Journal of Physical Chemistry B 117(38), 10974-86 (2013).

[35] van Oort, B., van Grondelle, R., and van Stokkum, I. H. M., "A Hidden State in Light-Harvesting Complex II Revealed By Multipulse Spectroscopy.," The Journal of Physical Chemistry B 119(16), 5184-93 (2015).

[36] Krüger, T. P., Ilioaia, C., Johnson, M. P., Ruban, A. V., Papagiannakis, E., Horton, P., and van Grondelle, R., "Controlled Disorder in Plant Light-Harvesting Complex II Explains Its Photoprotective Role," Biophysical Journal 102(11), 2669-2676 (2012).

[37] Nußberger, S., Dörr, K., Wang, D. N., and Kühlbrandt, W., "Lipid-protein Interactions in Crystals of Plant Lightharvesting Complex," Journal of Molecular Biology 234(2), 347-356 (1993).

[38] Liguori, N., Periole, X., Marrink, S. J., and Croce, R., "From light-harvesting to photoprotection: structural basis of the dynamic switch of the major antenna complex of plants (LHCII).," Scientific reports 5, 15661 (2015).

[39] Seddon, A. M., Curnow, P., and Booth, P. J., "Membrane proteins, lipids and detergents: not just a soap opera," Biochimica et Biophysica Acta (BBA) - Biomembranes 1666(1-2), 105-117 (2004). 